\title{
Multifilament carbon fibre tool electrodes in micro EDM-evaluation of process performance based on influence of input parameters
}

\author{
Anna Trych-Wildner ${ }^{1} \cdot$ Krzysztof Wildner $^{1}$
}

Received: 6 October 2016 / Accepted: 11 January 2017 / Published online: 27 January 2017

(C) The Author(s) 2017. This article is published with open access at Springerlink.com

\begin{abstract}
The aim of the study was to verify the possibility of application of multifilament carbon fibre electrodes for electrical discharge machining process. In the article, the novel type of tool electrode in form of a rod that is composed of carbon fibres was proposed. The performed analysis taking into account the influence of input parameter from $\mathrm{RC}$ generator like voltage, capacitance and resistance along with evaluation of the process with this novel type of electrodes was considered. The manufacturing technique was assessed by analysing the geometry parameters of machined holes like diameters and maximum depth. The detailed analysis and quantitative values of the impact of each of the input factors were presented and response surfaces according to the experimental methodology were obtained. The key efficiency parameter which was expressed by material removal rate (MRR) was also calculated and compared with data for other materials used as tool electrodes that were presented previously in literature. The comparison study proved that with multifilament carbon fibre electrodes the comparable range of MRR parameter in similar electro-erosive conditions can be obtained. Thus, they can be considered as complementary material for tool electrode purposes.
\end{abstract}

Keywords Carbon fibres $\cdot$ MicroEDM $\cdot$ Multifilament electrodes · Novel tool electrodes

Anna Trych-Wildner

A.Trych@mchtr.pw.edu.pl

1 Institute of Metrology and Biomedical Engineering, Faculty of Mechatronics, Warsaw University of Technology, ul. sw. Andrzeja Boboli 8, 02-525 Warszawa, Poland

\section{Introduction}

Electrical discharge machining (EDM) is well known manufacturing technique and its continuous industrial usage enforces permanent improvements to achieve desired performance and to understand better physical phenomena associated with the process $[1,2,8,10,16]$. In many cases to obtain better machining output and lower tool electrode wear new materials are tested $[7,11,12,15,17,19,22$, $26,27]$. Usually, in most applications, standard electrodes made of graphite, copper or other metals are considered [3, 5]. It is because of their high melting temperature and good thermal and electrical conductivity [3, 20]. Sometimes some changes to the standard process are proposed, like sequential machining using different manufacturing techniques or hybrid processes joining at least two manufacturing techniques simultaneously $[4,14,21]$.

When selecting the proper material for electrodes, one has to take into account not only conductivity, which is essential for electrodes in EDM, but also other features, like machinability and availability of the material along with thermal properties, mainly because of the thermal character of the process $[24,25]$.

Single carbon fibres that have been considered as electrode material in $[11,12,17,19]$ have above-mentioned properties at acceptable level and can be used in electroerosive conditions. In this study, however, the new approach to the use of carbon fibres as tool electrodes is presented. The electrodes in a form of multifilament rods are proposed and examined. They can be considered as similar material to previously tested single carbon fibres $[11,12$, 17, 19] but their form should enhance the process output. The form of the electrode can also serve as a link between micro and macro features of machined shapes as 


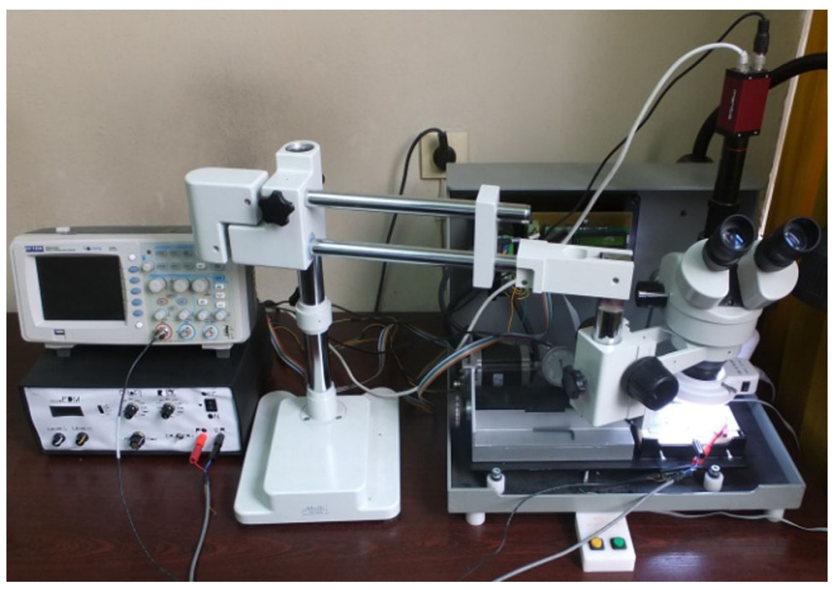

Fig. 1 Experimental setup (RC generator on the left)

it is possible to chose appropriate size of the tool electrode by gathering right number of single fibres to create desired tool dimension. Although the wear of single carbon fibre electrodes was noticeable, in a sense of linear shortening of a tool, what was reported in [17], it still enabled to perform the machining [17]. Multifilament electrodes with higher conductivity due to larger cross-section ( $\mu \mathrm{m}^{2}$ - single fibre vs. $\mathrm{mm}^{2}$-multifilament rod) should significantly change the wear of the tool and give also a possibility of manufacturing structures in wider range of diameter.

The aim of the paper was to analyse the influence of input parameters on the process performance and the evaluation of the key geometry parameters of machined holes and the process efficiency. Such approach with multifilament tool extends possibility of using this new material in function of tool electrode to manufacture wider range of shapes.

At first, the new electrodes are described with all experimental procedures and the applied methodology is also introduced. Then, the performance of multifilament electrodes is shown and quantitative description of the influence of input parameters on the depth of machined shapes, diameter and MRR is presented. Finally, comparison study of material removal rate (MRR) for different tool materials is discussed.

\section{Experimental setup and materials}

\subsection{Experimental setup}

The experiments performed in this study were conducted using the special experimental setup for micro electrical discharge machining (Fig. 1). The key components are an $\mathrm{RC}$ generator for $\mu \mathrm{EDM}$, a drive system and a microcontroller. The generator can be additionally equipped with an external inductance, resistance and capacitance to expand the possibilities of applied energies during the machining. The setup has also a camera to monitor the process and an oscilloscope. The parameters like resistance, capacitance and voltage can be chosen from range of $2 \mathrm{k} \Omega$ to $1 \mathrm{M} \Omega$, $2 \mathrm{pF}$ to $1000 \mathrm{pF}$ and 0 to $150 \mathrm{~V}$, respectively. The generator has also an adjustable level of drop voltage threshold which helps in detecting the short-circuit voltage level.

The proper detection of short-circuit enables the drive system to perform a retraction of an electrode. The levels of the drop voltage can vary from 0 -full short-circuit-to the maximum value given from the generator in each trail. Depending on the experiment, this parameter allows to continue forward movement up to the full short-circuit or to almost no discharge when the voltage is chosen at a very high level close to the given value from the generator. The example of voltage and current waveforms which represent single discharges is presented in Fig. 2.

Further details of the experimental setup and the design process can be consulted in [18].
Fig. 2 Example of voltage (upper line) and current (lower line) waveforms during $\mu \mathrm{EDM}$

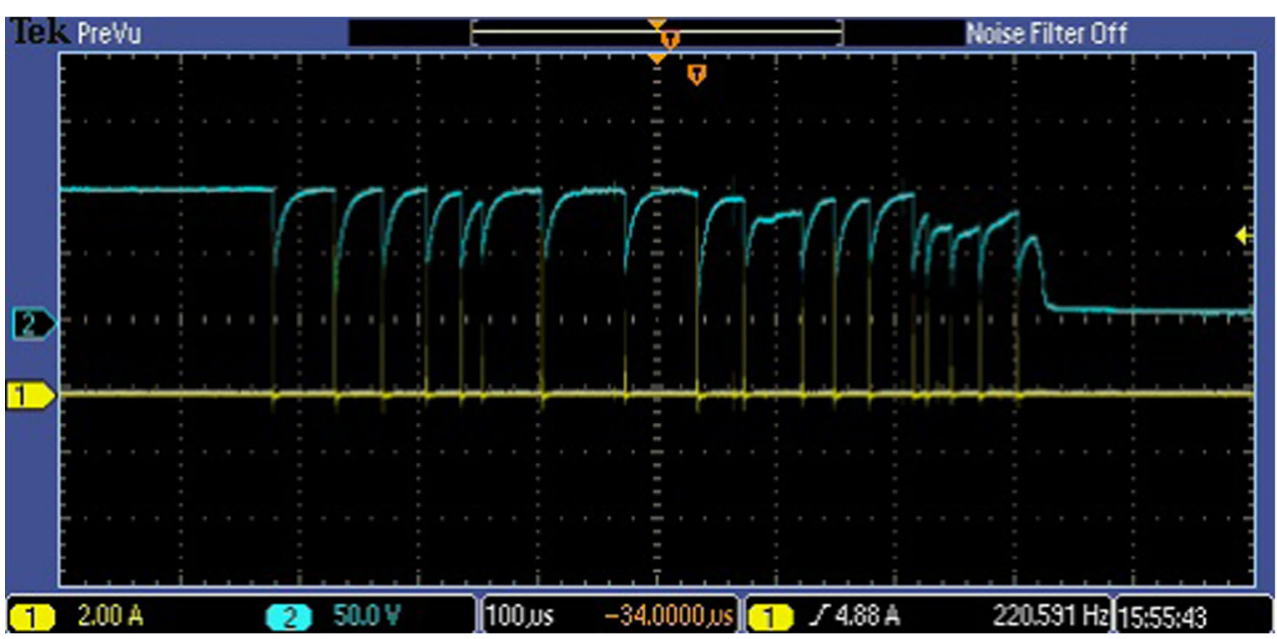




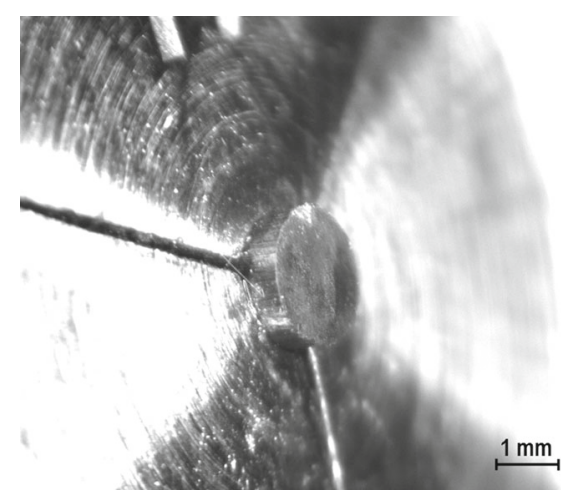

Fig. 3 Example of an electrode (diameter of $1.5 \mathrm{~mm}$ )

\subsection{Materials}

The electrodes used in this study were made of carbon fibres gathered in rods. The diameter of electrodes in experiments was $1.5 \mathrm{~mm}$ (Fig. 3).

The lengths of electrodes were from 13.5 to $17.8 \mathrm{~mm}$. Single carbon fibres create multifilament electrode that is coated with tiny layer of polymer that holds all the fibres together ensuring the shape of the tool. The material for tools was obtained from Carbon Fibres Australia supplier. Since novel form of tool electrode contains about a few thousand of fibres, their conductivity should be higher in comparison with single fibre, due to larger cross-section. The form is obtained in process of pultrusion which enables to achieve the material with constant cross-section being covered with layer of polymer. In case of the material used in experiments, it contained $95 \%$ of unidirectional carbon fibre running longitudinally (http://www.carbonfiber.com. $\mathrm{au}$ ). As the carbon fibre are usually consider for constructions that requires strength, typically mechanical properties are given. Such properties are gathered in Table 1 based on supplier data. Additionally, the calculated conductivity of the sample used in experiments for the length of about $15.5 \mathrm{~mm}$ is also presented. This is crucial property for tool electrodes. However, the measurement performed on the rod is an approximate value mainly because the poor surface

Table 1 Typical properties of carbon fibres based on data from supplier

\begin{tabular}{ll}
\hline Property & Value \\
\hline Tensile strength & $3530 \mathrm{MPa}$ \\
Tensile modulus & $230 \mathrm{GPa}$ \\
Elongation & $1.5 \%$ \\
Density & $1.76 \mathrm{~g} / \mathrm{cm}^{3}$ \\
Conductivity $^{\mathrm{a}}$ & $674.7 \mathrm{~S} / \mathrm{m}$ \\
\hline
\end{tabular}

${ }^{\mathrm{a} O w n}$ experimental data
Table 2 Values of input parameters used in the experiments

\begin{tabular}{lll}
\hline Variable & High level & Low level \\
\hline Voltage & $130 \mathrm{~V}$ & $100 \mathrm{~V}$ \\
Capacitance & $1000 \mathrm{pF}$ & $100 \mathrm{pF}$ \\
Resistance & $100 \mathrm{k} \Omega$ & $10 \mathrm{k} \Omega$ \\
\hline
\end{tabular}

contact of the probes to the tested sample and the presence of the sizing (polymer coating) which is an insulator.

Material used as a specimen was a gauge block made of steel (100Cr6) with smooth surface to better identify even slightly machined areas. As a dielectric fluid a cosmetic kerosene was used.

\section{Experimental procedures}

To find the influence of input parameters on the depth of obtained cavities the experiments were performed according to the experiment methodology by choosing the two-level three-factors plan with transformation of examined factors [9]. The plan was chosen because it allows to perform the experiments with small number of tests that can give the first approach to the new material before considering wider analysis. The plan assumes the high and low level of each of the input parameters (Table 2). The values of each level were chosen based on preliminary tests in which the process with single carbon fibres was able to be performed [11, 12, 17, 19].

Then, the plan foreseen a coding procedure. The higher level and lower level of input factors are assigned to plus and minus signs, respectively, to form a table to run the tests [9]. The variables were coded at different levels according to Table 2 and collected in Table 3.

$\mathrm{X} 1, \mathrm{X} 2$ and $\mathrm{X} 3$ represents voltage $(U)$, capacitance $(C)$ and resistance $(R)$, respectively, and the experiments were executed according to the Table 3 with high or low value of voltage, capacitance and resistance.

Table 3 Coded input parameters

\begin{tabular}{llll}
\hline Run & X1 & X2 & X3 \\
\hline 1 & + & + & + \\
2 & + & - & - \\
3 & - & + & - \\
4 & - & - & + \\
5 & + & + & - \\
6 & + & - & + \\
7 & - & + & + \\
8 & - & - & - \\
\hline
\end{tabular}




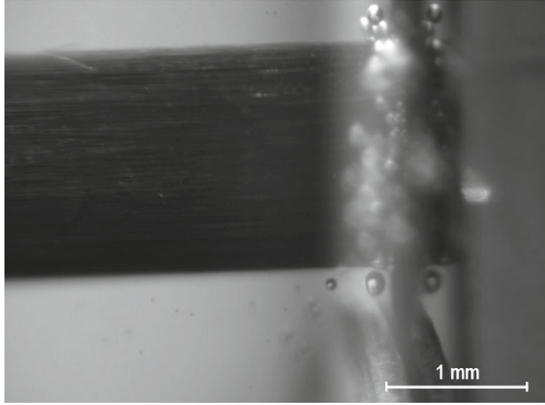

Fig. 4 Snapshot taken during $\mu \mathrm{EDM}$

Other input factors like feed motion and type of dielectric fluid were the same in all experiments. The time of machining was $60 \mathrm{~min}$ for each trial. The drop voltage threshold was assumed to be at about $70 \%$ of input voltage. The drop at this level starts the retraction movement of electrode and thereby gives time to prepare another full discharge. Figure 4 presents a snapshot taken during $\mu \mathrm{EDM}$. The bubbles formed after the discharges in the dielectric fluid in the working area are clearly visible.

\section{General observations and shape analysis of machined holes}

After carrying out the experiments, the obtained cavities were photographed and their shapes were analysed. Obtained cavities differ from each other according to input parameters for each trial what is presented in Figs. 5 and 6 . Figure 5 (before cleaning) shows well the sludge from dielectric fluid around each hole.

This is an effect of thermal phenomena which are present during discharges and decompose dielectric fluid-kerosene. The sludge can be easily removed from the surface mechanically or in an ultrasonic cleaner (Fig. 6).

As it is presented in Fig. 6 (after cleaning), not every trial had the input parameters at levels enabling to achieve holes that are the mirror image of the electrode what is usually the case in electroerosive conditions. It is clearly seen in Figs. 5 and 6 that the cavities 1, 7, 4 and 6 were not machined well in a sense of achieving the target shape. The electrode, even though the time of machining was $60 \mathrm{~min}$, did not immerse fully producing the mirror image of the tool.

The diameters of the holes which are the mirror images of the tool were similar to the diameter of electrodes. For the test 2,3,5 and 8, average diameter measured in perpendicular directions were as follows: $1.521,1.538,1.568$ and 1.503 (in $\mathrm{mm}$ ) with standard deviation respectively: 0.068 , $0.069,0.070$ and 0.067 . Analysing the data in which the target shape was achieved, it can be assumed that, in provided conditions, the oversize error (the difference between the diameter of the tool and the obtained hole) caused by side working gap, always present in EDM, was very small and did not exceed $5 \%$. The oversize of cavities for all the experiments is presented in Fig. 7.

The oversize error was calculated as the difference between tool diameter and the diameter of the resulting cavity. The same approach was applied to those cavities which did not achieved target shape. In this case, however, the average diameters were much smaller than the tool electrode. Thus, negative values from Fig. 7 correspond to these tests and indicates also how little area was affected by electroerosive conditions in these cases. The higher the number of negative values of the oversize error in Fig. 7 the smaller area (calculated from average diameter measured in four different directions) was obtained.

For each hole, a profile, with the use of Taylor Hobson Talysurf 10 profilometer, was recorded and the maximum depth was measured. Figure 8 represents the example of the profile for hole No. 3 from the table of experiments.

It is visible that only one side of hole is machined deeply, the other one is only slightly affected by $\mu$ EDM process. It could be caused by inclination of electrode or better machining conditions occurring on one side. The electrode which was used to machine the hole No. 3 was then also measured and photographed. Figures 9 and 10 shows the profiles of the tool and face end of electrode, respectively.

The wear of tool electrodes is difficult to estimate by means of measuring the length with the accuracy of one decimal place. Comparing to the data that were given in [17], the linear wear of single fiber would have resulted after the time of machining applied during this study in noticeable wear of a few millimeter. So the wear expressed as the lost in

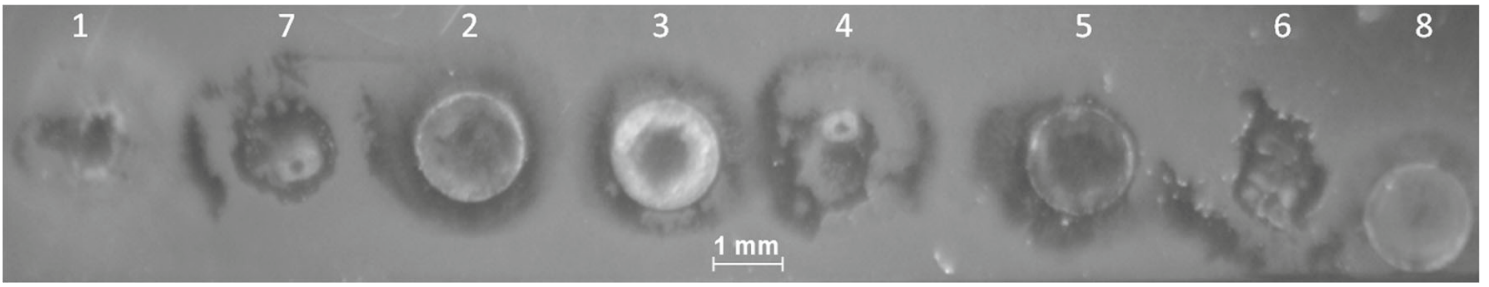

Fig. 5 Cavities obtained during experiments—-before cleaning (numbers correspond with Table 2. 


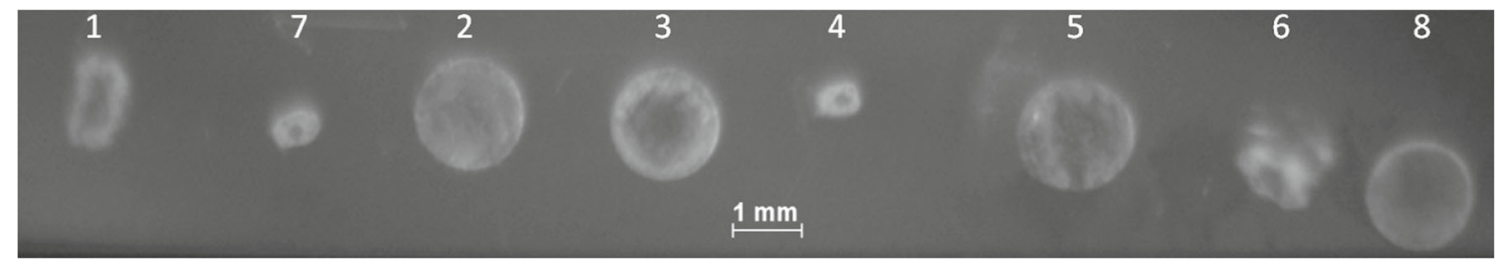

Fig. 6 Cavities obtained during experiments—after cleaning (numbers correspond with Table 2)

length per unit time for the multifilament electrode in such comparison is very low considering 60 min of machining.

\section{Influence of input parameters on the chosen geometry indicators of machined holes}

Micro-EDM is electro-thermal process which depends mostly on the key parameters responsible for energy of single pulse that are voltage and capacitance given from the $\mathrm{RC}$ generator during the machining. To verify the influence of these parameters, the planned experiment was preformed. The capacitance connected with the charging and discharging in the process and the frequency of pulses in RC-circuit will be influencing strongly the process performance. As well the voltage supplied from the generator, responsible for the gap voltage, also contributes to the energy of single discharge. The resistance together with capacitance in case of RC-circuit is responsible for the time in which capacitor will be charged and therefore it contributes to the frequency of the pulses.

To compute the quantitative impact of input parameters on the indicators like depth, diameter of machined cavities and efficiency of the process expressed by MRR the procedure from the planned experiment was performed according to the test methodology [9]. This methodology assumes the execution of test according to the Table 3 from Section 3. Data obtained from the experiments enable to receive the

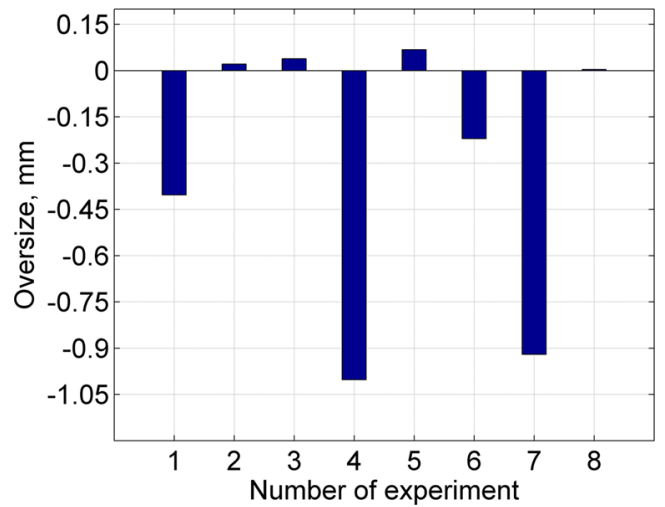

Fig. 7 Oversize of machined cavities regression model of the influence of tested input parameters $(U, C, R)$ on the resultant parameter (depth, diameter and MRR). It is expressed by the following formula:

$Y=b_{0}+b_{1} X_{1}+b_{2} X_{2}+b_{3} X_{3}$

where $Y$ is the resultant parameter for example depth, $b$ with index from 0 to 3 are regression coefficients that are expressed by Formula (3) and $X$ with index from 1 to 3 are input parameters in the coded form that are obtained from the Formula (2).

The coding procedure is expressed in the following way:

$X_{i}=\frac{2(\ln A-\ln H L)}{\ln H L-\ln L L}+1$

where $\mathrm{HL}$ is value of high level and LL is value of low level of respective input factors taken from the Table 2 and $A$ represents the considered factor $U, C$ or $R . X_{i}$ represents the coded input factor needed for Formula (1). The regression coefficients are calculated from the obtained data for all the experiments in the following way:

$b_{i}=\frac{1}{N} \sum_{j=1}^{N} x_{i j} y_{j}$

where $N$ is a number of experiments, in considered case 8 according to Table 3, $i$ is the index from 0 to 3 as in the Formula (1), $j$ represents following runs in the experiments from 1 to $8, x$ represents following values from the Table 3

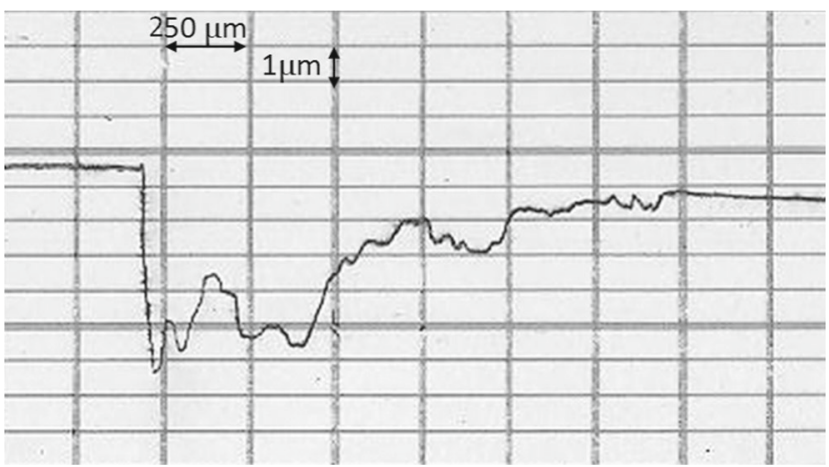

Fig. 8 Example of the profile of the hole 3 
Fig. 9 Example of surface of the electrode 3 after machining

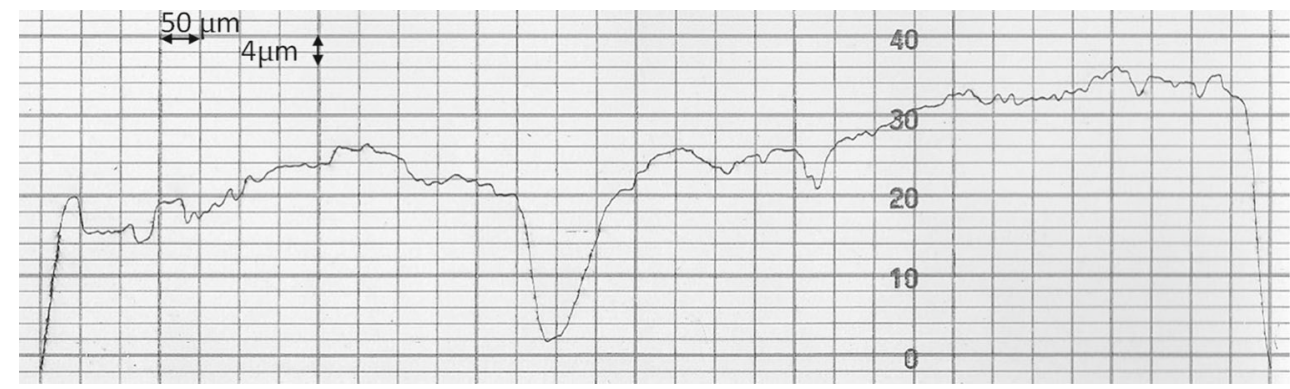

(it is either -1 or +1 depending on the sign in the table and $x_{0}$ is +1 ) and $y$ represents the measured resultant parameter form the experiment, for example depth.

After ordering and substituting Eqs. (2) and (3) to Eq. (1), the relationship of the chosen geometry indicators versus input parameters can be found. Such procedure is applied to the measured geometry parameters of machined holesdepth, diameter of machined cavities and efficiency of the process expressed by MRR.

\subsection{Diameter analysis}

After calculation and simplification of the Formula (1), the relationship describing the effect of the examined input parameters was obtained. For the mean diameter of the machined holes it is expressed as follows:

$Y=0.02 \frac{U^{1.54} C^{0.01}}{R^{0.28}}$

The diameter for the calculations was measured for each hole as the mean from four measurements performed in four different directions.

According to Formula (4), the response surfaces could be obtained (Figs. 11, 12 and 13).

As it is shown in Figs. 11 and 13, the most significant factor influencing the diameter of obtained holes is voltage given from the generator. It has also the highest value of exponent in Formula (4). The capacitance, responsible

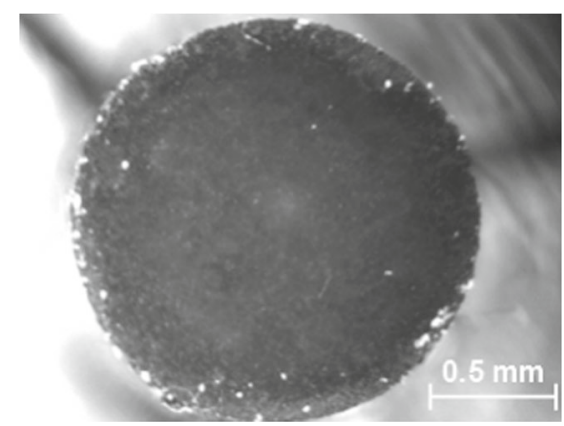

Fig. 10 Photograph of the face end of the electrode 3 after machining for the energy as well as the voltage in case of RC generators, has very low value of exponent. According to [9], the low values of exponent typically below 0.1 could be neglected. As Figs. 12 and 13 show, the increase of the capacitance does not visibly change the response surfaces. The resistance which is inversely proportional to frequency of discharges in case of RC generators has the exponent value more than five times smaller than for the voltage. However, it can be noticed in Figs. 11 and 12 that the rise of analysed mean diameter for the resistance is present.

The methodology of the experiments adapted for the analysis also allows to consider the influence of input energy calculated as the energy provided from the generator given by the formula as follows:

$E=\frac{C \cdot(0.75 U)^{2}}{2}$

where $E$ is the energy of single discharges, $C$ is the capacitance and $U$ is the voltage given from the generator. For calculation, 0.75 maximum value of voltage from generator as the threshold value when the discharges occur was taken.

Both parameters responsible for energy affect the diameter of the holes. It is seen in Fig. 14 that for the lower level of $\mathrm{R}$ (lower graph) the energy of discharges is influencing the analysed parameter with visible proportional dependence. For those trials, the target shape of the holes was obtained. Figure 14 (upper graph) shows the trials in which target shape was not observed.

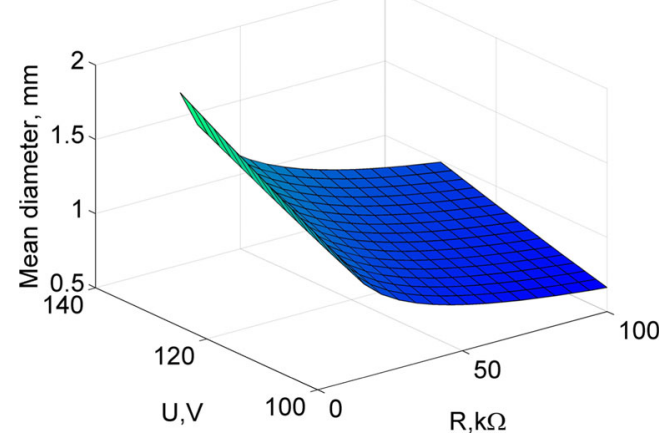

Fig. 11 Response surface with constant value $C=100 \mathrm{pF}$ 


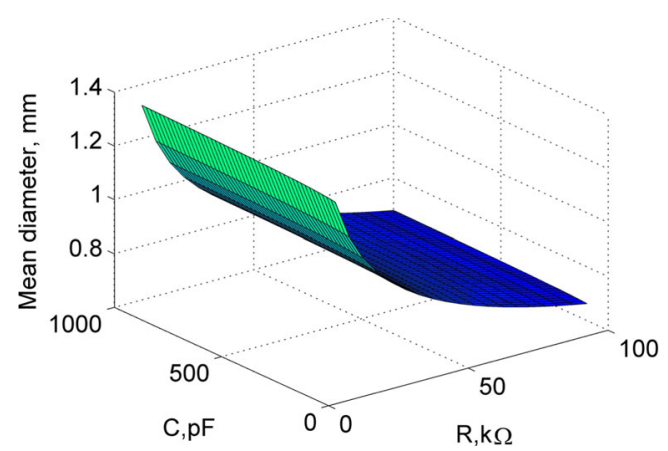

Fig. 12 Response surface with constant value $V=100 \mathrm{~V}$

As the Formula (4) takes into account all the manufactured cavities, those which obtained target shape and those without desired shape, it almost eliminated the capacitance from the consideration (very low exponent value). For the presented data, the very important factor was the resistance that for lower value enables to achieve target shape whereas for higher applied value it was not possible even though the parameters responsible for energy were high resulting in high energy of single discharge. Thus, even though the resistance do not contribute to the energy directly according to theoretical Formula (5), it supplies to the process the suitable parameters for the frequency in which the energy through the working gap can act. In analysed case, too high resistance results in not achieving the target shape of the cavity. Thus, the most favourable parameters should be chosen as these with the lowest resistance. It cannot be eliminated fully because arcing phenomenon can occur instead of generating sparks [23]. However, in the considered case, the lower $R(10 \mathrm{k} \Omega)$ was sufficient to achieve the mirror shape of tool electrode.

\subsection{Depth analysis}

The same analysis of the data was employed for the depth of cavities. The considered parameter was the maximum depth recorded from the holes profiles. Following the same approach as in Section 5.1, the formula that describes

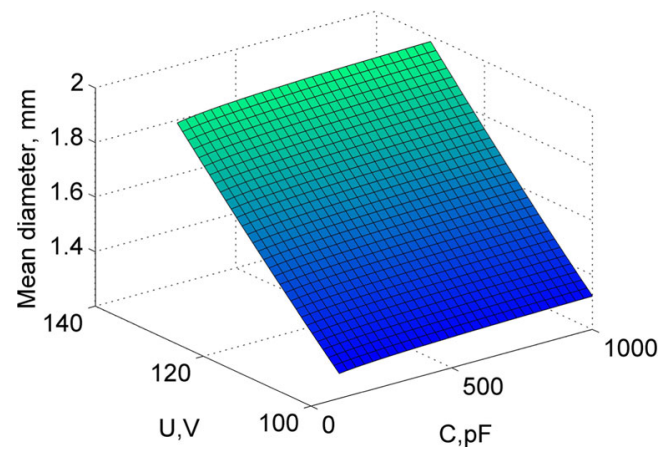

Fig. 13 Response surface with constant value $R=10 \mathrm{k} \Omega$
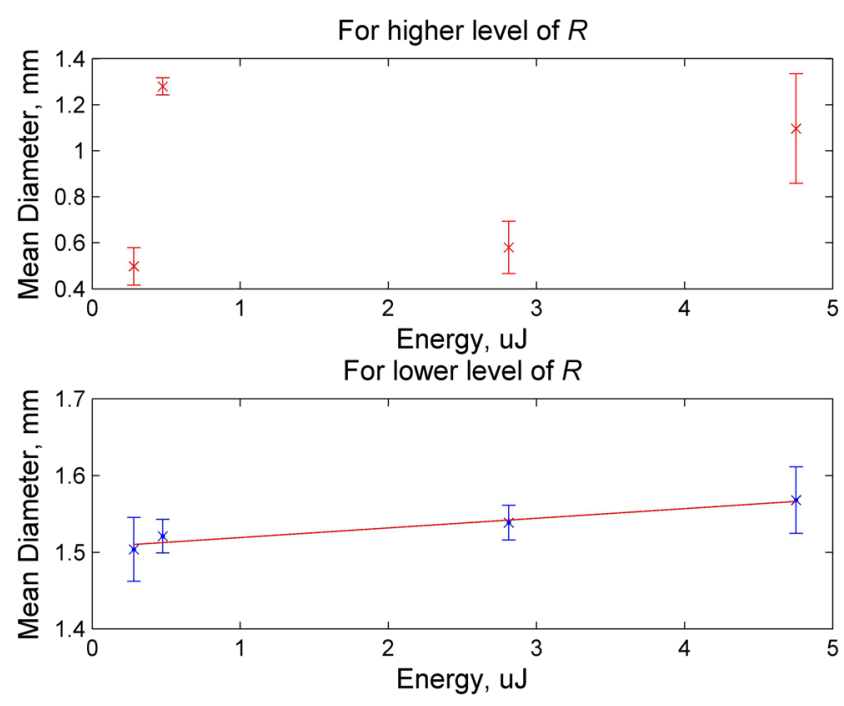

Fig. 14 Relationship of energy of single discharges and mean diameter of hole for lower and higher resistance value

the influence of applied input factors was obtained. The relationship describing the effect of the examined input parameters was as follows:

$Y=0.28 \frac{U^{0.77} C^{0.31}}{R^{0.82}}$

The exponent value in Formula (6) characterising voltage $U$ is almost 2.5 times higher than the exponent for capacitance C. Thus, the contribution of this parameter in obtaining the deep holes in the case of multifilament carbon fibres electrodes is significant. The rise in depth values can be observed in Figs. 15 and 16.

For RC generator, which was used in the experiments, the frequency of discharges is inversely proportional to $R$ and as well $C$ values, and in the same way, it should affect the depth of the machined holes. However, $C$ parameter which has still lower values of exponent in comparison with voltage cannot be neglected. It also corresponds with input energy, thus its effect on the process is twofold. The changes in response

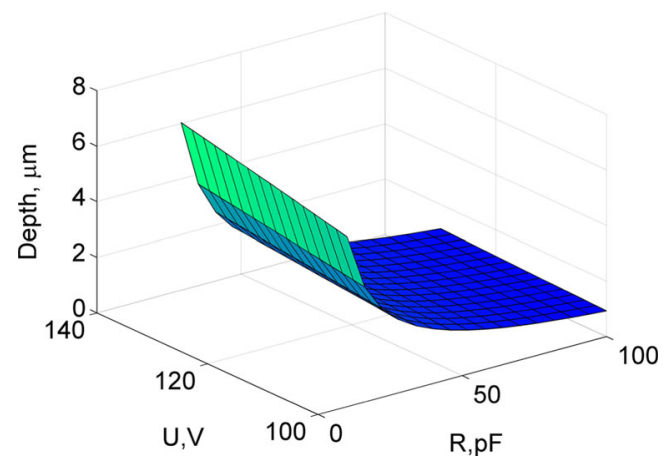

Fig. 15 Response surface with constant value $C=100 \mathrm{pF}$ 


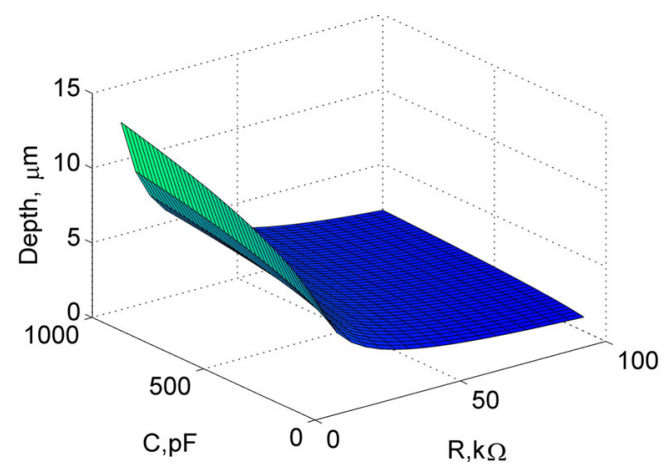

Fig. 16 Response surface with constant value $U=100 \mathrm{~V}$

surfaces with the change in $C$ parameter can be observed in Figs. 16 and 17.

In case of depth, all the analysed input parameters in contrast to analysis of diameter in Section 5.1 are significant. The highest value of exponent has the resistance that significantly changes the shape of the response surfaces in Figs. 15 and 16.

From the closer analysis of Fig. 18, it can be noticed that the maximum depth of machined shapes can be up to six times deeper for the low value of resistance than for the high value. Whereas differences in other input parameters do not provide such a high change in performance measured here by the depth. Therefore, similarly as in the Section 5.1, minimising the resistance, for obtaining in this case the maximum depth, is preferable because it enhances the frequency of discharges. When considering micro-EDM in which the energies are in range of $\mu \mathrm{J}$ providing higher value of resistance results in lower depth and as in previous section was the case, not achieving the target shape.

\subsection{MRR analysis}

Material removal rate (MRR) is a resultant parameter that takes into account the geometry of the machined shape: in analysed case the diameter and the maximum depth of the

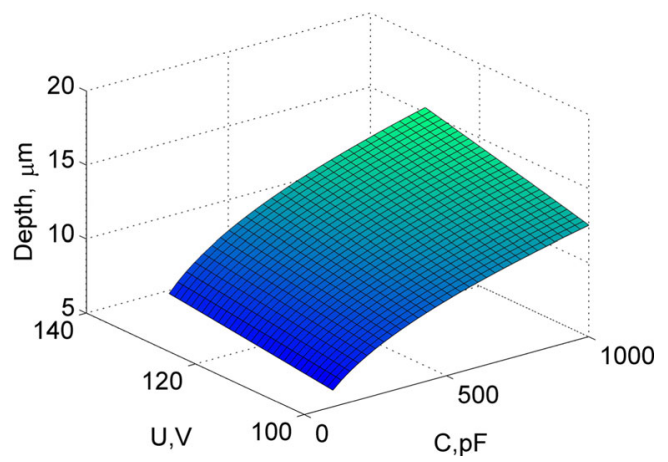

Fig. 17 Response surface with constant value $R=10 \mathrm{k} \Omega$

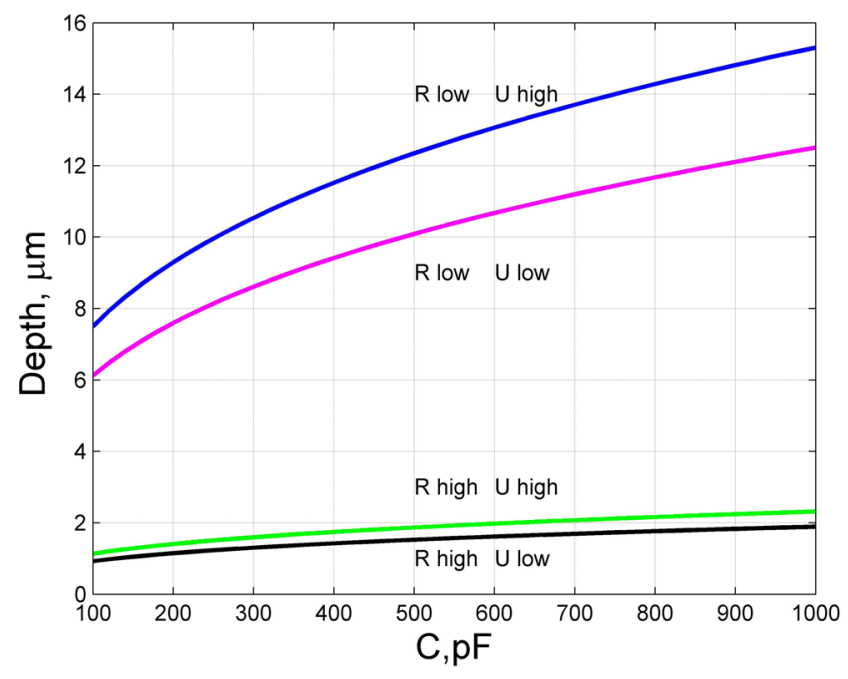

Fig. 18 Depth vs. capacitance for different values of resistance and voltage

holes and also the time of machining. It expresses the process efficiency as volume of material removed per time unit. It is worth to note the maximum measured depth was taken into account when calculating MRR. As Figs. 19, 20 and 21 show, the character of the surface response are similar to those in the previous section. However, the influence of the voltage is being more noticeable for MRR (Fig. 21).

All the response surfaces are based on the following formula obtained for MRR:

$Y=0.000001 \frac{U^{3.85} C^{0.32}}{R^{1.38}}$

Considering the capacitance, it has still similar level of exponent as for the depth analysis. However, in case of MRR, both voltage and resistance exponents are higher. The voltage has about five times higher value than for the relationship of depth and 2.5 times higher in comparison with the mean diameter. Thus, for efficiency of the manufacturing process those two parameters are the most important. As it was noted for this parameter the maximum depth was considered for calculating the MRR. This output parameter is

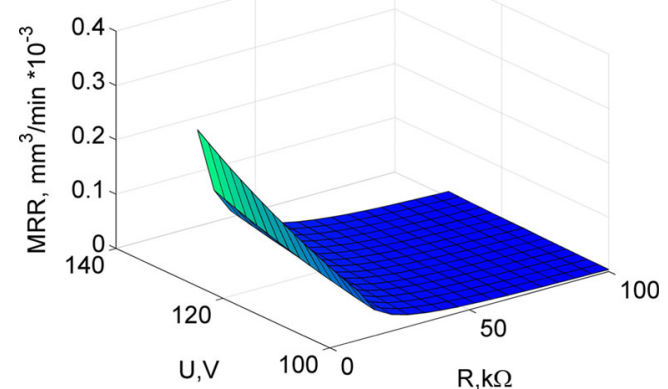

Fig. 19 Response surface with constant capacitance $C=100 \mathrm{pF}$ 


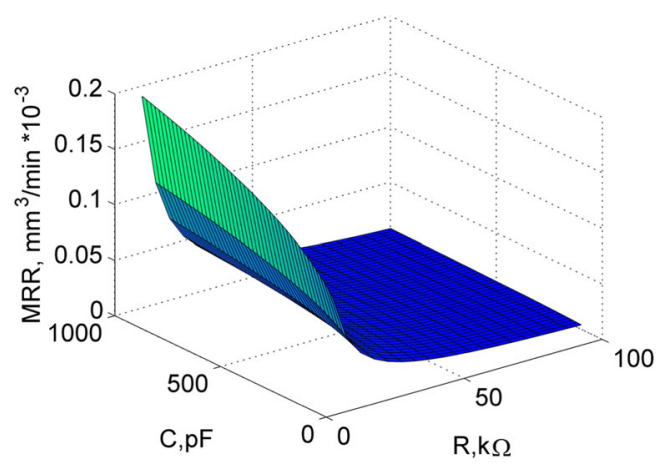

Fig. 20 Response surface with constant voltage $U=100 \mathrm{~V}$

therefore strongly influenced by the maximum depth which has high values for those trial for which the diameter also takes high values.

Both the diameter and the depth are present when calculating the MRR (volume per time unit) thus they naturally increase this parameter. As the volume takes multiple geometrical parameters into account, therefore, exponents' values become higher in Formula (7) and the exponents corresponding with the diameter put into the formula the greatest contribution. Further, the MRR is connected with time of machining and also being inversely proportional to the frequency of discharges, $R$ responsible for this parameter becomes the important factor in the formula. Considering all this, the efficiency of the process expressed by MRR strongly depends on all the factors taken into account. Whereas only geometrical parameters are less influenced when comparing the exponents. However, when considering RC generator the analysed resultant parameters do not follow fully the trends as in Section 5.1 in which $C$ exponent was very low. With low frequency and not fully charged capacitor, what may happen with RC-circuit, it will not discharge the maximum energy, rather there will be variation in the discharge energy [6]. Thus, it may disturb the result compared with what is theoretically expected and lower or even eliminate the exponent for some parameters as it was the case in Section 5.1.

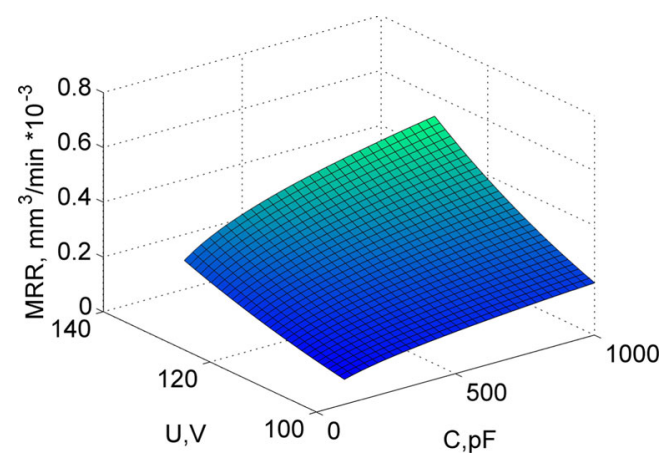

Fig. 21 Response surface with constant resistance $R=10 \mathrm{k} \Omega$

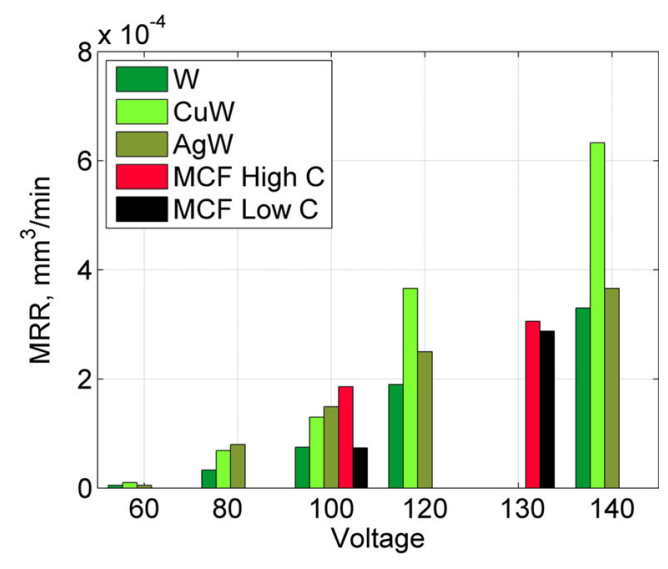

Fig. 22 Comparison of MRR of multifilament carbon fibre electrodes with other materials

\subsection{Comparison study of MRR with other electrode materials}

When analysing the proposed electrodes with other materials, similar tendencies can be observed $[5,6]$. For the comparison study, the data from the literature were examined [5]. The authors tested three types of electrodes made of $\mathrm{W}, \mathrm{CuW}$ and $\mathrm{AgW}$. The gap voltage starting from 60 up to $140 \mathrm{~V}$ significantly increases the MRR. Figure 22, in case of multifilament carbon fibres, shows similar data. The next parameter introduced by authors strongly influencing the MRR was capacitance starting with stray capacitance up to $47 \mathrm{pF}$. For the carbon fibres, the two tested values were 100 and $1000 \mathrm{pF}$ which also increased the MRR. However, for higher values of voltage it is less noticeable, whereas for lower voltage the change in capacitance results in more than two times change in MRR (Fig. 23).

The comparison made between the data from [5] (Fig. 22) suggests that the MRR achieved in similar conditions for novel multifilament electrodes are at the same order of magnitude as for compared materials. Authors in [5] examined

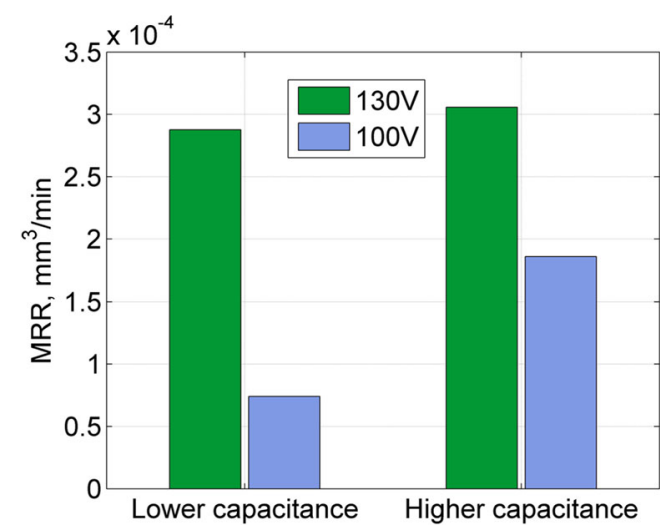

Fig. 23 MRR of multifilament carbon fibres electrodes for chosen tests 
three electrode materials machining microhole in tungsten carbide workpiece. As it is presented in Fig. 22, for which data of all three materials are shown, namely for $\mathrm{W}, \mathrm{AgW}$ and $\mathrm{CuW}$, for the constant values of resistance and capacitance, the change only in voltage involves the increase of MRR. Comparing the values of this parameter for novel electrodes, there were considered two values of voltage. The level of MRR for $100 \mathrm{~V}$ was similar to the literature data. Only lower values of capacitance decreased MRR but still it can be compared to the value achieved for $\mathrm{W}$ electrode. For $130 \mathrm{~V}$, there was no data for comparison but MRR for $140 \mathrm{~V}$, in the next trial, shows that for $\mathrm{W}$ and $\mathrm{AgW}$ electrodes even for higher value of voltage the analysed parameter takes comparable level. In a straight comparison of different electrode materials the multifilament carbon fibres electrodes can be regarded as similar to compared electrodes in terms of MRR. However, the considered data were obtained for electrodes with diameter of $500 \mu \mathrm{m}$ whereas multifilament electrodes were three times thicker. The second important issue is that for calculation of analysed parameter maximum depth was taken into account which slightly overstated the MRR. Another issue to consider is the time of machining which in case of novel electrodes was the total time of experiment. It does not exclude the time consumed by short-circuits or the retraction movement during which the machining is not taking place. However, considering all this it cannot be stated that novel electrodes are inferior to these taken for examination, but still at comparable level.

Additionally, the compared experiment had the value of resistance fixed to $1 \mathrm{k} \Omega$ [5]. Therefore, it provided the higher frequency of discharges than in the experiments with carbon fibres. Thus, the data obtained for carbon fibres may result in slightly lower values of MRR than for $\mathrm{W}, \mathrm{CuW}$ and $\mathrm{AgW}$. So taking the maximum depth for calculation is partially compensated by lower frequency during the experiments. However, the key indicator of MRR proves to be in the same range of order as considered materials.

\section{Conclusions and summary}

The paper proposed the micro electrical discharge machining process involving the usage of a novel electrode made of carbon fibres gathered to form a rod electrode. The experiments proved the possible application of multifilament carbon fibre electrodes in $\mu$ EDM. The rod electrodes were more resistant to process condition than single carbon fibres due to larger cross-sections. The tests with single carbon fibres could last only in range of tens of second up to couple of minutes until significant reduction in length prevented them from further machining [17]. An application of multifilament rod electrodes increases the possible time of machining in comparison with single fibres. The wear of the multifilament electrodes in such condition was difficult to estimate. However, analysing what was reported in [17] where the shortening of single fibre was even up to $5 \mathrm{~mm}$, multifilament tools were not reduced in length in such a way even during longer machining. Nonetheless, in favourable conditions manufacturing of holes on the workpiece surface which are being the mirror image of the tool could be achieved.

In this study, the effect of input parameters on depth of holes obtained in $\mu$ EDM process with carbon fibres multifilament electrodes was examined. The relationship gives preliminary information of quantitative impact of individual input factors on the output value-maximum depth, diameter or MRR. The influence of individual input parameters was described with formulas. According to the test methodology [9], the exponent value gives information on how much each factor can affect; in considered case, the geometrical parameters of the manufactured holes and only values below 0.1 could be neglected from the formulas. Thus, it was found that the resistance, being responsible for frequency of discharges and having the highest value of exponent, is the most significant factor for the maximum depth. Whereas for diameter and resulting parameter of MRR, the voltage was the most influencing factor in the analysed range of input parameters. The shape of holes machined with considered electrodes were mirror image of the tool in tests $2,3,5$ and 8 in which the resistance provided from the RC generator was at the lower level. Also, the maximum depth for these tests had the highest values. Thus, for maximising these geometrical parameters of manufactured holes, the resistance should be at very low level. The results of the test with RC generator are with accordance with the results shown previously in the literature for other electrodes like brass [13] or tungsten [6].

The conducted experiments proved that a bunch of carbon fibres gathered in the rod electrode can effectively work as a tool electrode. They introduced also quantitative data about the performance of the process measured as the maximum depth, the diameter of obtained holes and MRR. The MRR parameter compared with other materials proves that examined electrodes can be considered as comparable with $\mathrm{W}, \mathrm{AgW}$ and $\mathrm{CuW}$ electrodes in terms of efficiency of the process and can be regarded as potential complementary electrode material for EDM.

It should be also possible to design such electrodes into other shapes and their diameters can vary according to the number of single fibres gather into a rod. Further development of the technology with multifilament carbon fibres electrodes need to consider methods for the process optimisation for maximising the depth or achieving other desired geometrical parameters of the machined shapes by expanding the range of tested parameters. Also quantitative 
data about the tool wear should be obtained to verify the qualitative observations from this study.

Finally, it should be noted that rising prices of different materials enforces searching for substitute or complementary materials that could fulfil the function of tool electrodes effectively, at least in certain applications. Thus, based on the presented study, multifilament carbon fibres, as their resemblance in terms of MRR to other electrodes materials was shown, should be regarded as complementary tool material for existing ones.

Acknowledgments This work has been partially supported by the European Union in the framework of the European Social Fund through the Warsaw University of Technology Development Programme, realised by the Center for Advanced Studies, Warsaw University of Technology and also by statutory funds from the Faculty of Mechatronics, Warsaw University of Technology.

Open Access This article is distributed under the terms of the Creative Commons Attribution 4.0 International License (http:// creativecommons.org/licenses/by/4.0/), which permits unrestricted use, distribution, and reproduction in any medium, provided you give appropriate credit to the original author(s) and the source, provide a link to the Creative Commons license, and indicate if changes were made.

\section{References}

1. Chiou AH, Tsao CC, Hsu CY (2015) A study of the machining characteristics of micro EDM, milling and its improvement by electrode coating. Int J Adv Manuf Technol 78:1857-1864

2. Dimov SS et al. (2006) A roadmapping study in multi-material micro manufacture. In: 2nd International conference on multimaterial micro manufacture, Grenoble

3. D'Urso G, Maccarini G, Ravasio C (2015) Influence of electrode material in micro-EDM drilling of stainless steel and tungsten carbide. Int J Adv Manuf Technol

4. Golabczak A, Swiecik R (2013) Assessment of energy consumption and internal stresses in surface layer in the abrasive electro-discharge grinding (AEDG) process. Defect and Diffusion Forum, Trans Tech Publications 334:89-96

5. Jahan M, Wong Y, Rahman M (2009) A study on the finefinish die-sinking micro-EDM of tungsten carbide using different electrode materials. J Mater Process Technol 209(8):3956-3967

6. Jahan M, Wong Y, Rahman M (2009) A study on the quality micro-hole machining of tungsten carbide by micro-EDM process using transistor and rc-type pulse generator. J Mater Process Technol 209(4):1706-1716

7. Khanra AK, Sarkar BR, Bhattacharya B, Pathak LC, Godkhindi MM (2007) Performance of ZrB2-cu composite as an EDM electrode. J Mater Process Technol 183(1):122-126

8. Kitamura T, Kunieda M, Abe K (2015) Observation of relationship between bubbles and discharge locations in EDM using transparent electrodes. Precis Eng 40:26-32
9. Korzynski M (2006) Experiment methodology, Warszawa

10. Kozak J, Rozenek M, Dabrowski L (2003) Study of electrical discharge machining using powder-suspended working media, Dabrowski, L.: Study of electrical discharge machining using powder-suspended working media. P I Mech Eng B-J Eng 217(11):1597-1602

11. Kudla L (2009) Micro electrical, discharge machining using carbon fibres as tool electrodes I:105-108

12. Kudla L, Trych A (2010) Characterisation and experimental testing of the properties of carbon fibres in respect of their application as tool electrodes for micro EDM. In: 16th international symposium on electromachining Shanghai, China

13. Natarajan N, Suresh P (2015) Experimental investigations on the microhole machining of 304 stainless steel by micro-EDM process using rc-type pulse generator. Int $\mathbf{J}$ Adv Manuf Technol 77(9):1741-1750

14. Skoczypiec S, Ruszaj A (2014) A sequential electrochemicalelectrodischarge process for micropart manufacturing. Prec Eng 38(3):680-690

15. Song KY, Park MS, Chu CN (2013) EDM turning using a strip electrode. J Mater Process Technol 213(9):1495-1500

16. Teimouri R, Baseri H (2012) Improvement of dry EDM process characteristics using artificial soft computing methodologies. Prod Eng Res Devel 6:493-504

17. Trych A (2013) Further study of carbon fibres electrodes in micro electrical discharge machining. Procedia CIRP 6:309-313

18. Trych A, Markiewicz S (2012) Idea of the experimental stand for micro-electrical discharge machining using carbon fibres as tool electrodes. J Mach Eng 12(2):105-110

19. Trych-Wildner A, Kudla L (2016) Can carbon fibres work as tool electrodes in micro electrical discharge machining? J Micromech Microeng 26(7):075,007

20. Tsai HC, Yan BH, Huang FY (2003) EDM Performance of $\mathrm{Cr} / \mathrm{Cu}-$ based composite electrodes. Int J Mach Tool Manu 43(3):245252

21. Uhlmann E, Domingos DC (2016) Investigations on vibrationassisted EDM-machining of seal slots in high-temperature resistant materials for turbine components part II. Procedia CIRP 42:334-339

22. Uhlmann E, Roehner M (2008) Investigations on reduction of tool electrode wear in micro-EDM using novel electrode materials. J Manufacturing Sci Technol 1(2):92-96

23. Wong Y, Rahman M, Lim H, Han H, Ravi N (2003) Investigation of micro-EDM material removal characteristics using single rc-pulse discharges. J Mater Process Technol 140(1-3):303-307. Proceedings of the 6th Asia Pacific Conference on materials Processing

24. Yeo S, Kurnia W, Tan P (2008) Critical assessment and numerical comparison of electro-thermal models in EDM. J Mater Process Technol 203(1-3):241-251

25. Yeo SH, Kurnia W, Tan PC (2007) Electro-thermal modelling of anode and cathode in micro-EDM. J Phys D Appl Phys 40(8):2513

26. Yoo HK, Kwon WT, Kang S (2014) Development of a new electrode for micro-electrical discharge machining (EDM) using Ti(c, N)-based cermet. Int J Precis Eng Manuf 15(4):609-616

27. Zaw HM, Fuh JYH, Nee AYC, Lu L (1999) Formation of a new EDM electrode material using sintering techniques. J Mater Process 89-90:182-186 